\title{
Technologies for metal recycling from electrical - electronic wastes
}

\author{
Toi Trung Tran*, Chinh Thi Vu, Nhung Thi Pham \\ Hanoi University of Mining and Geology, Hanoi, Vietnam
}

\section{ARTICLE INFO}

Article history:

Received 06 ${ }^{\text {th }}$ Feb. 2021

Revised 15th May 2021

Accepted $12^{\text {th }}$ June 2021

\section{Keywords:}

Electric - electronic equipment,

Recycling technology,

Separation processes,

Waste.

\section{ABSTRACT}

Wastes from electrical - electronic equipment (WEEE) are of huge concerns worldwide. With the decreasing life cycle of most electrical electronic equipment (EEE), the WEEE growth rate is fast, about $3 \div 5 \%$ annually. This is considered as the fastest growing waste stream among municipal wastes. WEEE contains, in addition to plastic, glass etc., high contents of base metals and precious metals. They are regarded as potential secondary resources of metals in addition to primary resources of metals from metallic ores. Over the past decades, many metal recycling technologies from WEEE have been developed including physical separation processes and chemical extraction processes. This article summarizes the characteristics, recycling principles, separation processes and optimal operating conditions of the world's most currently common technologies for metal recycling from WEEE. So that one can derive new directions for metal recycling from electrical electronic wastes in the Vietnam case.

Copyright @ 2021 Hanoi University of Mining and Geology. All rights reserved.

${ }^{*}$ Corresponding author

E - mail: trantrungtoi@humg.edu.vn

DOI: 10.46326/JMES.2021.62(3b).07 


\title{
Tạp chí Khoa học Kỹ thuật Mỏ - Địa chất
}

Trang điện tử: http://tapchi.humg.edu.vn

\section{Công nghệ tái chế kim loại từ rác thải điện - điện tử}

\author{
Trần Trung Tới * Vũ Thị Chinh, Phạm Thị Nhung \\ Trường Đại học Mỏ - Địa chất, Hà Nội, Việt Nam
}

THÔNG TIN BÀI BÁO

Quá trình:

Nhận bài 06/02/2021

Sửa xong $15 / 5 / 2021$

Chấp nhận đăng 12/6/2021

\section{Tù̀ khóa:}

Chất thải,

Công nghệ tái chế,

Quy trình tuyển tách,

Thiết bị điện - điện tử.

\section{TÓM TẮT}

Chất thải tạo ra tù các thiết bị điện - điện tử (WEEE) là mối quan tâm lớn trên toàn thế giới. Hiênn nay, hầu hết các thiết bi điện - điện tưu (EEE) có vòng đời ngày càng giảm, nên lượng WEEE ngày càng tăng, với tốc độ tăng hàng năm khoảng $3 \div 5 \%$. Đây được coi là dòng chất thải có tốc độ phát sinh nhanh nhất so với các loại chất thải khác ở đồ thị. WEEE có các thành phần ngoài nhựa, thủy tinh, ... còn có hàm lượng cao các kim loại co bản và kim loại quý. Do đó, WEEE được coi là nguồn tài nguyên thứ sinh tiềm năng để tái chế kim loại. Trong thập kỷ qua, đã có nhiều quy trình công nghệ tái chế kim loại tù̀ WEEE được đề cập, tù các quy trình tuyển vật lý đến các quy trình công nghệ hóa học. Bài báo viết tống hợp về đặc điểm, nguyên tắc tái chế, quy trình phân tách và các thông số vận hành tối ưu của các công nghê tái chế kim loại tù̀ WEEE hiện đang sử dụng phổ biến trên thế giới. Tù̀ đó, nhận định hướng đi mới trong công cuộc tái chế kim loại từ chất thải điện - điện tử ở Việt Nam.

C 2021 Trường Đại học Mỏ - Địa chất. Tất cả các quyền được bảo đảm.

\section{Mở đầu}

Đại dịch COVID - 19 đã ảnh hưởng đáng kể đến hoạt động giao tiếp hàng ngày của con người. Nhu cầu kết nối ngày càng tăng thông qua các hoạt động trực tuyến từ xa đã tạo ra xu hướng tiêu dùng mói, đồng thời làm dấy lên lo ngại về sự gia tăng số lượng các thiết bị điện - điện tử (EEE). Bên cạnh đó, tuổi thọ của các thiết bị điện - điện tử ngày càng giảm so với trước đây. Vì vậy, lượng phát sinh chất thải từ thiết bị điện, điện tử (WEEE) ngày càng tăng cao (Vanessa và nnk., 2020). Năm 2019, thế giới đã tạo ra 53,6 triệu tấn chất thải điện tử, trung bình là

\footnotetext{
*Tác giả liên hệ

E- mail: trantrungtoi@humg.edu.vn DOI: 10.46326/JMES.2021.62(3b).07
}

7,3kg/người. Lượng WEEE phát sinh trên toàn cầu đã tăng 9,2 triệu tấn kể từ năm 2014 và dự kiến sẽ tăng lên 74,7 triệu tấn vào năm 2030 - gần như tăng gấp đôi chỉ sau 16 năm. Lượng rác thải điện tử ngày càng tăng do tỷ lệ tiêu thụ các thiết bị điện - điện tử cao hơn, tuổi thọ của các EEE ngắn hơn và ít lựa chọn sửa chữa. Châu Á phát sinh lượng WEEE cao nhất vào năm 2019 ở mức 24,9 triệu tấn, tiếp theo là châu Mỹ (13,1 triệu tấn) và châu Âu (12 triệu tấn), trong khi châu Phi và châu Đại Dương phát sinh lần lượt là 2,9 triệu tấn và 0,7 triệu tấn. Châu Âu đứng đầu thế giới về lượng rác thải điện tử phát sinh trên đầu người, với $16,2 \mathrm{~kg} /$ người. Châu Đại Dương đứng thứ hai $(16,1 \mathrm{~kg} / \mathrm{người})$, tiếp theo là châu Mỹ (13,3 kg/người), trong khi châu Á và châu Phi chỉ tạo ra lần lượt là $5,6 \mathrm{~kg}$ và $2,5 \mathrm{~kg} /$ người. (Vanessa và nnk., 2020). 


\section{2. Đặc điểm rác thải điện, điện tử}

Trong các thiết bị điện - điện tử có thể có tới hơn 1000 chất khác nhau, bao gồm các chất nguy hại (chì, thủy ngân, cadimi, asen...) và các chất không nguy hại. Rất khó để đưa ra thành phần chung cho toàn bộ dòng chất thải điện - điện tử. Tuy nhiên, hầu hết các nghiên cứu đều chia các thành phần vật liệu trong chất thải điện - điện tử thành năm nhóm: kim loại đen, kim loại màu, nhựa, thủy tinh và các thành phần khác như Hình 1 (Huy., 2017).
Hầu hết các thiết bị điện - điện tử thải đều có bản mạch in (PCBs), đây là đối tượng được quan tâm rất lớn trong lĩnh vực tái chế vì thành phần vật liệu có trong nó rất phong phú và có giá trị cao. Thành phần trung bình của bản mạch in gồm có khoảng 40\% trọng lượng kim loại, 30\% trọng lượng nhựa và $30 \%$ trọng lượng là vật liệu gốm sứ. Trong đó, đồng là kim loại chiếm hàm lượng cao nhất $10 \div 26,8 \%$ về trọng lượng và một hàm lượng không nhỏ, bạc chiếm từ $110 \div 3301$ ppm, vàng chiếm $80 \div 1000$ ppm, paladin chiếm từ $10 \div 294$ ppm và bạch kim chiếm từ $4,6 \div 30$ ppm (Bảng 1 ) (Paul., 2010).

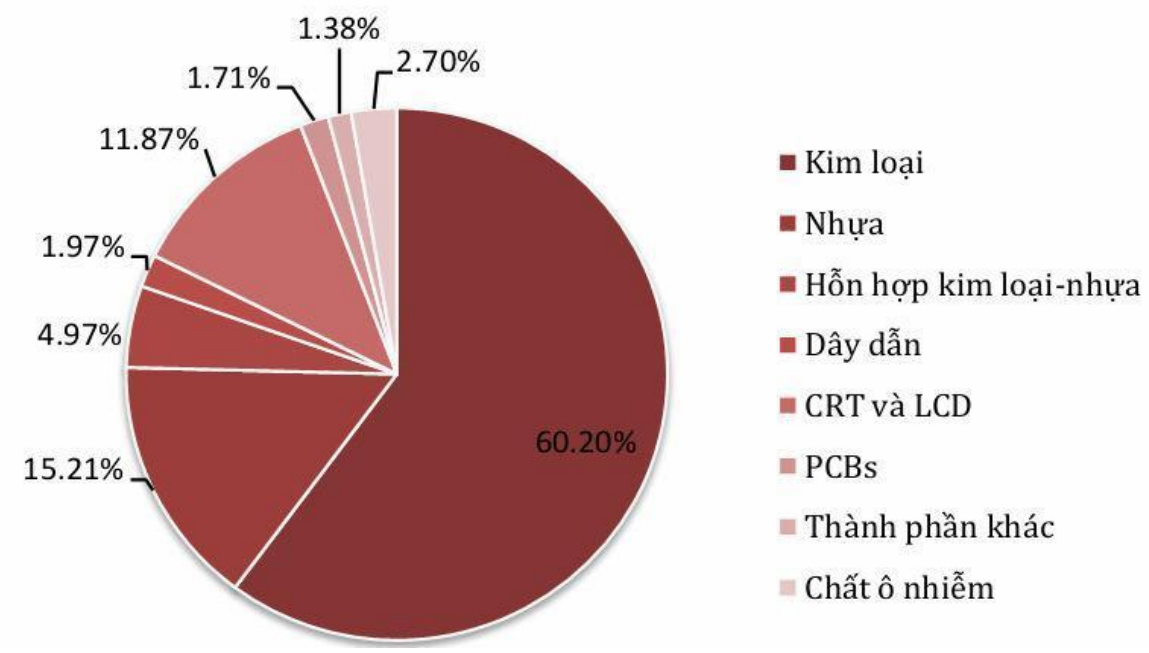

Hình 1. Thành phần vật liệu trong thiết bị điện và điện tử thải.

Bảng 1. Thành phần bảng mạch in PCBs (Paul., 2010).

\begin{tabular}{|c|c|c|c|}
\hline Vật liệu & Thành phần (\% trọng lượng) & Vật liệu & Thành phần $(\%$ trọng lượng) \\
\hline Kim loại & Khoảng $40 \%$ & Gốm sứ & Khoảng $30 \%$ \\
\hline $\mathrm{Cu}$ & $10 \div 26,8$ & $\mathrm{SiO}_{2}$ & $15 \div 414,86$ \\
\hline $\mathrm{Al}$ & $1,33 \div 4,78$ & $\mathrm{Al}_{2} \mathrm{O}_{3}$ & $6 \div 6,97$ \\
\hline $\mathrm{Pb}$ & $0,99 \div 4,19$ & Kiềm, oxit kiềm thổ & $6 \div 9,95$ \\
\hline $\mathrm{Zn}$ & $0,16 \div 2,17$ & Titan, mica,.. & 3 \\
\hline $\mathrm{Ni}$ & $0,28 \div 2,35$ & Nhựa & Khoảng $30 \%$ \\
\hline $\mathrm{Fe}$ & $1,22 \div 8,0$ & Polyethylen & $9,9 \div 16$ \\
\hline $\mathrm{Sn}$ & $1,0 \div 5,28$ & Polypropylen & 4,8 \\
\hline $\mathrm{Sb}$ & $0,06 \div 0,4$ & Polyeste & 4,8 \\
\hline $\mathrm{Au}$ & $80 \div 1000 \mathrm{ppm}$ & Epoxie & 4,8 \\
\hline $\mathrm{Pt}$ & $4,6 \div 30 \mathrm{ppm}$ & Polyvinyl clorua & 2,4 \\
\hline $\mathrm{Ag}$ & $110 \div 3301 \mathrm{ppm}$ & Polytetra fuoroethan & 2,4 \\
\hline $\mathrm{Pd}$ & $10 \div 294 \mathrm{ppm}$ & Nylon & 0,9 \\
\hline
\end{tabular}


Trong một nghiên cứu của Xue Wang và nnk. (2012) về tiềm năng và mức độ ưu tiên để thu hồi các kim loại có trong PCBs của chất thải điện tử cho thấy, $\mathrm{Au}$ và $\mathrm{Pd}$ đứng đầu bảng vì giá trị, xếp thứ 3 là $\mathrm{Cu}$, bởi vì sự kết hợp của giá trị và trọng lượng của Cu trong PCBs thải, Hình 2.

Thực chất của quá trình thu hồi kim loại trong rác thải điện tử là thu hồi đồng thô, trong đó có lẫn nhiều kim loại quý như vàng, bạc, paladi,... cũng như các tạp chất khác. Các kim loại hay tạp chất này

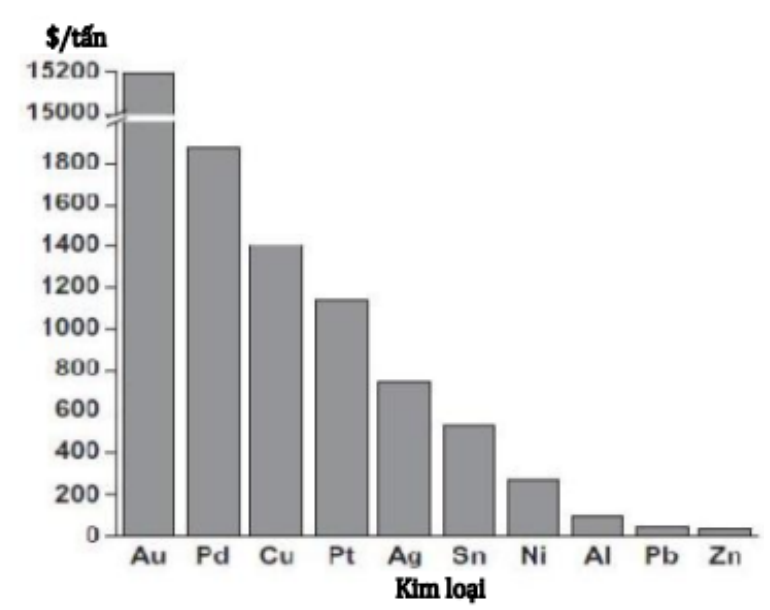

Hình 2. Nhóm 10 kim loại có giá trị được ưu tiên trong 1 tấn PCBs. (Xue và Gabrielle., 2012) sau đó được tách ra khỏi đồng bằng quá trình tinh luyện. Sơ đồ công nghệ tổng quát tái chế rác thải điện - điện tử được thể hiện ở Hình 3.

Như vậy, mặc dù WEEE chỉ chiếm $3 \div 5 \%$ trong chất thải rắn, nhưng sự đa dạng và hàm lượng các kim loại có giá trị lại lớn hơn nhiều so với các dạng chất thải khác (Li và nnk., 2015). Vì vậy, WEEE xứng đáng với tên gọi "đô thị mỏ”. Theo khảo sát của Cơ quan Bảo vệ Môi trường, Hoa Kỳ, so với sản xuất kim loại chính thì tái chế kim loại từ WEEE có những lợi thế đáng kể, chẳng hạn như ít chất thải thứ cấp hơn và tiêu thụ năng lượng thấp hơn. (He và nnk., 2006).

\section{Công nghệ tái chế kim loại từ WEEE trên thế giói}

\subsection{Tiền xử lý}

Để tái chế các kim loại có giá trị từ WEEE, trước tiên cần phải thực hiện bước tiền xử lý bằng phương pháp cơ học (Yoo và nnk., 2009). Các thành phần và thiết bị khác nhau có thể được tháo dỡ chọn lọc và tách thành nhiều phần khác nhau như kim loại (sắt, đồng, nhôm, magiê,...), nhựa, gốm, giấy, gố và các thiết bị như tụ điện, pin, ống hình, màn hình LCD và PCB (Antrekowitsch nnk., 2006).

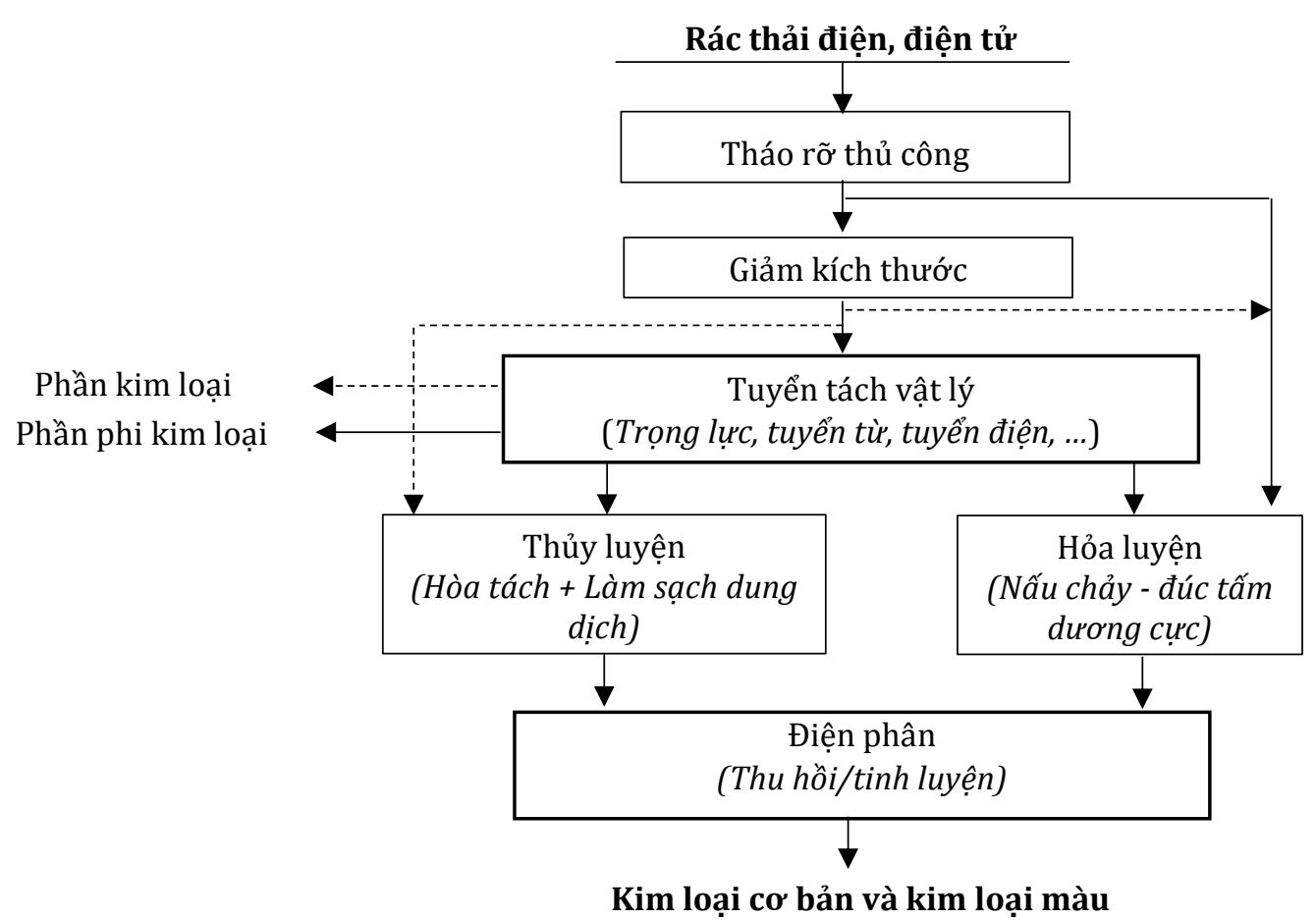

Hình 3. Lưu trình công nghệ tổng quát tái chế kim loại từ rác thải điện - điện tử (Phỏna theo Yazıcı và Deveci.. 2009). 
Các thành phần này có thể được tái sử dụng hoặc chuyển sang quy trình tái chế khác. Quá trình tháo dỡ cũng cho phép loại bỏ các thành phần độc hại và tách riêng trước các kim loại có giá trị, làm tăng tiềm năng kinh tế của chất thải và khả năng tái chế của chúng (Cui và Forssberg., 2003).

Sau khi loại bỏ và phân loại, các thành phần mang kim loại như PCBs sẽ được giảm kích thước trước khi tái chế kim loại. Máy cắt và máy nghiền búa được sử dụng để phân mảnh, nghiền, cắt hoặc xé chất thải (Dalrymple và nnk., 2007). Mức độ giảm kích thước phụ thuộc vào quy trình tách và thu hồi được sử dụng sau đó. Với hỏa luyện, chỉ cần vật liệu tương đối thô, còn quá trình phân tách vật lý và luyện kim thủy luyện cần nguyên liệu ở dạng mịn hơn (Duan và nnk., 2009). Theo nghiên cứu của Zhang và Forssberg (1997) cho thấy, đồng gần như giải phóng hoàn toàn khỏi phế liệu $\mathrm{PC}$ và $\mathrm{PCB}$ khi giảm kích thước dưới $2 \mathrm{~mm}$ và giải phóng kém hơn ở kích thước thô trên $2 \mathrm{~mm}$. Điều này là do sự liên kết của các chân đồng với nhựa và bao bọc các đoạn dây đồng trong chất dẻo.

\subsection{Phân tách vật lý}

WEEE chứa nhiều loại vật liệu có tính chất vật lý khác nhau như: khối lượng riêng, từ tính, độ dẫn điện,... (xem Bảng 2). Do đó, có thể dễ dàng tách riêng các kim loại từ các thành phần phi kim loại (tuyển trọng lực) và giữa các kim loại với nhau (tuyển từ, tuyển tĩnh điện, tuyển dòng xoáy) (Galbraith và Devereux., 2002; Zhang và Forssberg, 1997; Li và nnk.., 2004)

Các quy trình phân tách vật lý có vốn đầu tư và chi phí vận hành thấp. Một trong những hạn chế lớn của quy trình này là tổn thất kim loại có giá trị lên tới $10 \div 35 \%$. Lý do của những tổn thất này gồm việc giải phóng kim loại không đủ do sự liên kết mật thiết của các kim loại có giá trị với chất dẻo, tạo ra các hạt nhỏ $(<75 \mu \mathrm{m})$ trong quá trình giảm kích thước và sự kém hiệu quả của các quá trình tuyển để thu hồi kim loại từ các hạt mịn (Zhao và nnk, 2004). Sau khi xử lý cơ học/vật lý, các sản phẩm giàu kim loại được tiếp tục xử lý bằng các quy trình luyện kim để chiết tách kim loại cơ bản và kim loại quý.

Bảng 2. Tính chất vật lý của kim loại và nhựa có trong WEEE (Cui và Forssberg, 2003).

\begin{tabular}{|c|c|c|c|}
\hline Vật liệu & $\begin{array}{c}\text { Khối lượng riêng } \\
\left(\mathrm{g} / \mathrm{cm}^{3}\right)\end{array}$ & $\begin{array}{l}\text { Tính dẫn điện } \\
\left(10^{6} \mathrm{~m}^{-1} \Omega^{-1}\right)\end{array}$ & $\begin{array}{l}\text { Hệ số từ cảm } \\
\left(10^{6} \mathrm{~cm}^{3} \mathrm{~mol}^{-1}\right)\end{array}$ \\
\hline \multicolumn{4}{|l|}{ Kim loại } \\
\hline $\mathrm{Au}$ & 19,32 & 41,0 & Nghịch từ ( - 28) \\
\hline $\mathrm{Pd}$ & 12,02 & 9,50 & Thuận từ (+540) \\
\hline $\mathrm{Pb}$ & 11,40 & 5,00 & Nghịch từ ( - 23) \\
\hline $\mathrm{Ag}$ & 10,49 & 68,0 & Nghich từ $(-2,5)$ \\
\hline $\mathrm{Cu}$ & 8,93 & 59,0 & Nghịch tù̀ $(-5,46)$ \\
\hline Co & 8,90 & 17,2 & Sắt từ \\
\hline $\mathrm{Ni}$ & 8,90 & 12,50 & Sắt từ \\
\hline $\mathrm{Fe}$ & 7,86 & 9,33 & Sắt từ \\
\hline $\mathrm{Sn}$ & 7,30 & 9,17 & Thuận từ \\
\hline $\mathrm{Cr}$ & 7,19 & 7,74 & Thuận tù̀ (+167) \\
\hline $\mathrm{Al}$ & 2,70 & 37,7 & Thuận từ $(+16,5)$ \\
\hline Nhựa & & Điện trở suất $(\Omega . m)$ & \\
\hline Nhựa gia cố bằng sợi thủy tinh & $1,8 \div 2,0$ & $10^{12}$ & \\
\hline Nhựa polyme - este (PET, PBT) & $1,31 \div 1,39$ & $1 \div 1,4.10^{13}$ & \\
\hline Nhựa PVC & $1,16 \div 1,38$ & $10^{9} \div 2.10^{12}$ & \\
\hline Nhựa polyme - cabonat (PC) & 1,22 & $8,2.10^{14}$ & \\
\hline Nylon và nhựa PA & 1,14 & $10^{12}$ & \\
\hline Acrylonitrile (ABS) & 1,04 & $10^{14}$ & Nghịch từ \\
\hline Nhựa PS & 1,04 & $10^{14}$ & \\
\hline Nhựa PE & $0,91 \div 0,96$ & $10^{14}$ & \\
\hline Nhựa PP & 0,90 & $10^{15}$ & \\
\hline Chất đàn hồi & $0,85 \div 1,25$ & $10^{9} \div 10^{15}$ & \\
\hline
\end{tabular}




\subsection{Xử lý hóa}

\subsubsection{Phương pháp hỏa luyện}

Công nghệ luyện kim hỏa luyện được cải tiến để ứng dụng vào công nghệ tái chế và xử lý chất ô nhiễm. Đây là quá trình nấu chảy rác thải điện tử trong lò nhiệt độ cao. Trong quá trình này, đồng bị nấu chảy thành dạng lỏng và các kim loại có khả năng hòa tan trong đồng sẽ tan vào đồng nóng chảy Sản phẩm của quá trình là đồng thô có chứa một số kim loại khác như vàng, bạc,... Trong quá trình hỏa luyện, sắt và nhôm không bị hòa tan vào đồng mà đi vào xỉ. Rác thải điện tử có thể được tái chế trong lò cỡ nhỏ. Tuy nhiên, thông thường chúng được xử lý bằng phương pháp kết hợp với tinh quặng sunphua đồng để làm nguyên liệu trong các nhà máy luyện đồng lớn. Hiện nay, có bốn công ty đi đầu về xử lý rác thải điện tử để thu hồi đồng là Boliden, Xstrata Copper, Aurubis và Umicore; trong đó có 3 công ty của Châu Âu và một của Canada. Phương pháp Noranda là phương pháp tiêu biểu được sử dụng để thu hồi đồng từ rác thải điện tử. Thực chất của phương pháp này là việc trộn lẫn rác thải điện tử đã qua xử lý sơ bộ với tinh quặng sunphua đồng, hỗn hợp sau đó được sử dụng làm nguyên liệu trong quy trình sản xuất đồng kim loại từ quặng (Moskalyk and Alfantazi, 2003). Sơ đồ nguyên lý của phương pháp được thể hiện ở Hình 4.

Phương pháp hỏa luyện cho năng suất cao, đồng thời cho phép thu hồi được các kim loại quý trong rác thải điện tử. Tuy nhiên, quá trình này gặp phải một số hạn chế như: việc thu hồi sắt và nhôm là rất khó do bị oxi hóa trong quá trình nấu luyện và đi vào xỉ; sự hiện diện của chất chống cháy brôm trong nguyên liệu có thể dẫn đến sự hình thành chất điôxin; không thể xử lý thu hồi các loại vật liệu nhựa; chế độ công nghệ bị phức tạp hóa do thành phần nguyên liệu đầu vào không ổn định và vốn đầu tư thiết bị lớn.

\subsubsection{Phương pháp thủy luyện}

Công nghệ thủy luyện để xử lý rác thải có nhiều ưu điểm nổi bật so với quá trình hỏa luyện như: dễ điều chỉnh, có thể khống chế chính xác để đạt được mục tiêu đặt ra và tương đối ít ô nhiễm hơn so với công nghệ luyện kim hỏa luyện (Ghosh và nnk, 2015). Quy trình thủy luyện có thể chia làm ba công

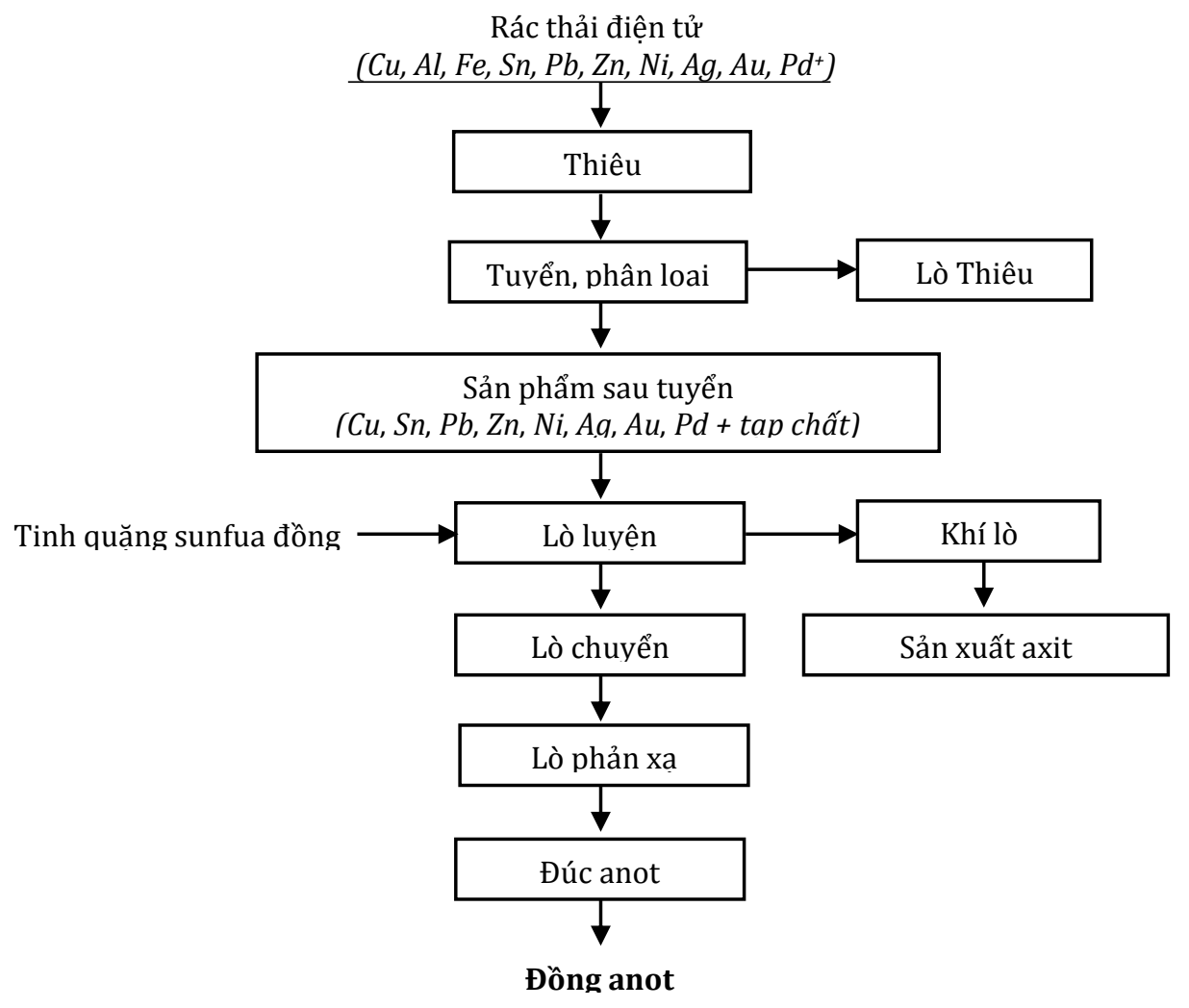

Hình 4. Sơ đồ nguyên lý phương pháp Noranda xử lý rác thải điện tử (Huy, 2017). 
đoạn chính: Hòa tách, xử lý dung dịch và thu hồi kim loại. Quá trình hòa tách thực hiện qua hai giai đoạn: giai đoạn đầu là hòa tách các kim loại cơ bản $(\mathrm{Cu}, \mathrm{Zn}, \mathrm{Pb}, \mathrm{Fe}, \ldots)$ và giai đoạn hai là hòa tách kim loại quý $(\mathrm{Au}, \mathrm{Ag}, \mathrm{Pd}, \ldots)$ từ bã còn lại của giai đoạn đầu (Hình 5).

Giai đoạn hòa tách thứ nhất sử dụng một số dung môi hòa tan hiệu quả và thân thiện đã được đề xuất, chẳng hạn như quá trình hòa tách clo (He và $\mathrm{Xu}, 2015)$, amoniac - ammonium (Sun và nnk., 2015) và giai đoạn hòa tách thứ hai không sử dụng xianua để hòa tách $\mathrm{Au}, \mathrm{Ag}$ (Akcil và nnk., 2015)

Dung môi hòa tách dựa trên clorua đã được phát triển và thực hiện ở quy mô thí điểm. Môi trường clorua $\left(\mathrm{HCl}-\mathrm{CuCl}_{2}-\mathrm{NaCl} ; \mathrm{H}_{2} \mathrm{SO}_{4}-\mathrm{CuSO}_{4}\right.$ $\mathrm{NaCl})$ để hòa tách đồng đã được sử dụng. Ion $\mathrm{Cu}^{2+}$ hoạt động như một chất oxy hóa đồng và hình thành sự ổn định của $\mathrm{Cu}^{+}$khi một phối tử thích hợp như ion clorua $(\mathrm{Cl}-)$ được thêm vào dung dịch sulfat
(Yazici và Deveci, 2015). Ion $\mathrm{Cu}^{2+}$ cũng có thể xuất hiện dưới dạng phức clo $\left(\mathrm{CuCl}_{n}{ }^{2-n}\right)$ trong dung dịch clorua (Yazici và Deveci, 2013). Các phản ứng được mô tả:

$$
\begin{gathered}
\mathrm{Cu}^{0}+\mathrm{Cu}^{2+}=2 \mathrm{Cu}^{+} \\
\mathrm{Cu}^{+}+\mathrm{nCl}=\mathrm{CuCl}_{n}^{1-n} \\
\mathrm{Cu}^{0+} \mathrm{Cu}^{2+}+2 \mathrm{Cl}=2 \mathrm{CuCl}_{\text {rắn }} \\
\mathrm{Cu}^{0}+\mathrm{Cu}^{2+}+4 \mathrm{Cl}=2 \mathrm{CuCl}_{2}
\end{gathered}
$$

Kết quả cho thấy khi dung môi ban đầu cho thêm $\mathrm{Cu}^{2+} \geq 79 \mathrm{mmol} / \mathrm{l}$ thì hiệu suất hòa tách đồng đạt 98\% sau 120 phút. Yazici và Deveci (2013, 2015) đã sử dụng hệ dung môi $\mathrm{H}_{2} \mathrm{SO}_{4}-\mathrm{CuSO}_{4}-\mathrm{NaCl}$ để hòa tách các kim loại từ chất thải PCB. Ảnh hưởng nồng độ ban đầu của đồng $(0,5 \div 7,5 \mathrm{~g} / \mathrm{l}$ $\left.\mathrm{Cu}^{2+}\right)$, clorua $(4,7 \div 46,6 \mathrm{~g} / \mathrm{l} \mathrm{Cl}-)$ và nhiệt độ $\left(20 \div 80^{\circ}\right.$ C) đến hiệu suất hòa tách đồng đã được khảo sát.

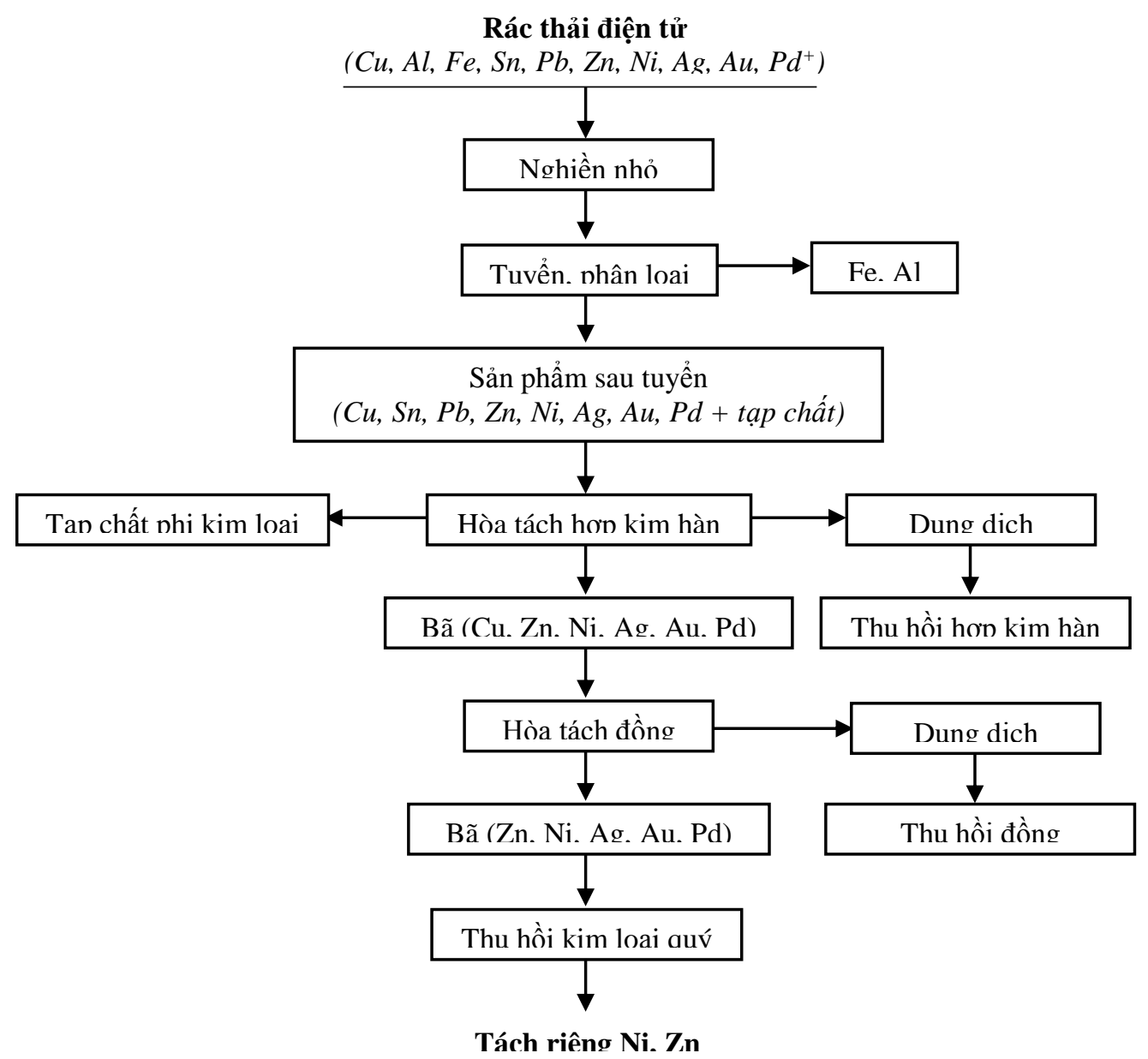

Hình 5. So đồ một quá trình thủy luyện xử lý rác thải điện, điện tử (Huy., 2017). 
Kết quả hiệu suất hòa tách đồng tăng cao khi nhiệt độ và nồng độ $\mathrm{Cu}^{2+}$ tăng lên. Tỷ lệ mol $\mathrm{Cl} / \mathrm{Cu}^{2+}$ rất quan trọng, vì $\mathrm{Cl}^{-}$và $\mathrm{Cu}^{2+}$ có tương tác mạnh mẽ trong quá trình hòa tách.

Dung môi hòa tách amoniac - amonium có tính ưu việt hơn về độ chọn lọc đối với đồng (Liu và nnk., 2009). Hệ thống amoniac - amoni sunfat và clorua đã được sử dụng để thu hồi $\mathrm{Cu}$ từ chất thải $\mathrm{PCB}$. Dung dịch muối amoniac - amoni chứa phức amin $\mathrm{Cu}$ (I) và $\mathrm{Cu}$ (II) được sử dụng làm dung môi hòa tách. Quá trình này bao gồm hai giai đoạn: hòa tách và làm sạch. Trong giai đoạn hòa tách, $\mathrm{Cu}$ trong chất thải PCB bị oxy hóa bởi Cu (II) để tạo thành ion phức $\mathrm{Cu}$ (I) ammine theo phản ứng:

$$
\mathrm{Cu}+\mathrm{Cu}\left(\mathrm{NH}_{3}\right)_{4}{ }^{2+}=2 \mathrm{Cu}\left(\mathrm{NH}_{3}\right)_{2}{ }^{+}
$$

Sự hình ion phức $\mathrm{Cu}\left(\mathrm{NH}_{3}\right)_{2}+$ làm tăng đáng kể hiệu suất hòa tan đồng (hiệu suất $>95 \%$ ). Dung dịch sau hòa tách được làm sạch và thu hồi đồng bằng phương pháp điện phân (Koyama và nnk., 2006). (Lim và nnk., 2013) đã sử dụng dung môi $\mathrm{NH}_{3} / \mathrm{NH}_{4} \mathrm{Cl}$ với chất oxi hóa $\mathrm{CuCl}_{2}$ để hòa tách $\mathrm{Cu}$ từ hợp kim thu được từ quá trình nấu chảy khử PCB của điện thoại di động. Kết quả, $98 \% \mathrm{Cu}$ hòa tan trong điều kiện: $2 \mathrm{kmol} / \mathrm{m}^{3} \mathrm{NH}_{4} \mathrm{Cl}$ và $5 \mathrm{kmol} / \mathrm{m}^{3}$ dung dịch $\mathrm{NH}_{3}, 0,1 \mathrm{kmol} / \mathrm{m}^{3} \mathrm{CuCl}_{2}$ ơ $^{3} 30^{\circ} \mathrm{C}$.

Đối với giai đoạn hòa tách thứ hai, để khắc phục những nhược điểm của việc hòa tách các kim loại quý bằng xyanua từ bã sau hòa tách kim loại cơ bản bằng clo hay amoniac, hiện nay sử dụng một số dung môi thân thiện môi trường, chẳng hạn như thiourea; thiocyanat và thiosulfate (Ha và nnk., 2010)

Thiourea $\left(\mathrm{NH}_{2}\right)_{2} \mathrm{CS}$ được sử dụng như một chất hòa tan $\mathrm{Au}$ từ WEEE, có hiệu suất hòa tách vàng cao và độc tính thấp (Gurung và nnk., 2013). Hiệu suất hòa tan Au phụ thuộc vào việc tối ưu hóa và kiểm soát cẩn thận độ $\mathrm{pH}$, thế oxy hóa khử, nồng độ thiourea $(>12 \mathrm{~g} / \mathrm{l})$ và thời gian hòa tách. Phản úng hòa tan vàng xảy ra theo phương trình:

$$
\mathrm{Au}+2 \mathrm{SC}\left(\mathrm{NH}_{2}\right)_{2}+\mathrm{Fe}^{3+}=\mathrm{Au}\left(\mathrm{SC}\left[\mathrm{NH}_{2}\right]^{2+}+\mathrm{Fe}^{2+}\right.
$$

Năm 2013, Behnamfard và cộng sự đã đề xuất công nghệ thủy luyện cải tiến để thu hồi có chọn lọc $\mathrm{Cu}, \mathrm{Ag}$, $\mathrm{Au}$ và $\mathrm{Pd}$ từ chất thải $\mathrm{PCB}$. Thứ nhất, qua hai bước hòa tách axit sunfuric liên tiếp với sự có mặt của $\mathrm{H}_{2} \mathrm{O}_{2}$ là chất oxi hóa, hơn $99 \%$ đồng được hòa tan. Bước thứ hai, hòa tách thiourea ở $\mathrm{pH}=1$ - 2 với $\mathrm{Fe}^{3+}$ là chất oxi hóa. Hiệu suất hòa tách đạt 85,76\% Au và $71,36 \%$ Ag. Quá trình tách $P d$ và $A u$ còn lại từ bã rắn ở bước thứ 3 được thực hiện trong hệ thống dung môi $\mathrm{NaClO}-\mathrm{HCl}-\mathrm{H}_{2} \mathrm{O}_{2}$. Khi đó $100 \% \mathrm{Pd}$ và $\mathrm{Au}$ tan trong dung dịch clorua và sau đó được kết tủa thu hồi bằng natri borohydrid $2 \mathrm{~g} / \mathrm{l}$ (Behnamfard và nnk., 2013)

Thiocyanat cũng là một dung môi hiệu quả cho hòa tan và tái chế vàng, bạc từ WEEE. Một đặc tính quan trọng của ion thiocyanat là nó có thể tạo thành các phức chất ổn định $\mathrm{Au}(\mathrm{SCN}) 2$ - và $\mathrm{Au}(\mathrm{SCN}) 4$ - (Li và nnk., 2012b). Trong dung dịch axit sunfuric với $\mathrm{Fe}^{3+}$ là chất oxi hóa, thiocyanat hòa tan vàng theo phản ứng:

$$
\begin{aligned}
& \mathrm{Au}+2 \mathrm{CSN}-+\mathrm{Fe}^{3+}=\mathrm{Au}(\mathrm{SCN})^{2-}+\mathrm{Fe}^{2+} \\
& \mathrm{Au}+4 \mathrm{CSN}-+\mathrm{Fe}^{3+}=\mathrm{Au}(\mathrm{SCN})^{4-}+\mathrm{Fe}^{2+}
\end{aligned}
$$

Tiếp theo các nghiên cứu trên, quá trình hòa tách vàng bằng phương pháp kết hợp thioureae thiocyanat, sứ dụng sắt ba sulfat làm chất oxy hóa (Yang và và nnk., 2011). Sự hòa tách vàng cao hơn so với dung dịch thiocyanate hoặc thiourea tương úng. Bởi trong hỗn hợp hai dung môi hòa tách này, vàng tan dạng phức chất hỗn hợp $\mathrm{Au}\left(\mathrm{SC}\left[\mathrm{NH}_{2}\right]_{2}\right)_{2} \mathrm{SCN}$.

\subsection{Phưong pháp vi sinh}

Bên cạnh quá trình hòa tách bằng hóa chất, xử lý rác thải điện tử bằng phương pháp vi sinh như một quy trình có chi phí thấp và thân thiện với môi trường cũng đã được chú ý trong nhũng năm gần đây. Quá trình vi sinh hòa tan kim loại từ WEEE thường được thực hiện bằng cách sử dụng các chủng vi khuẩn ưa axit để oxy hóa sắt hai $\mathrm{Fe}^{2+}$ lên $\mathrm{Fe}^{3+} \quad$ (Acidithiobacillus ferrooxidans và Leptospirillum ferrooxidans) dễ dàng. Muối sắt ba là một chất oxy hóa mạnh với thế khử tiêu chuẩn là 0,77 V nên dễ dàng oxy hóa các kim loại (ví dụ: $\mathrm{Cu}$ ) dẫn đến sự hòa tan của chúng theo phản ứng:

$$
\begin{gathered}
2 \mathrm{Fe}^{2+}+0.5 \mathrm{O}_{2}+2 \mathrm{H}^{+} \rightarrow 2 \mathrm{Fe}^{3+}+\mathrm{H}_{2} \mathrm{O} \text { (vi khuẩn) } \\
\mathrm{Fe}^{3+}+\mathrm{Cu}=\mathrm{Fe}^{2+}+\mathrm{Cu}^{2+} \text { (hóa học) }
\end{gathered}
$$

Duy trì môi trường axit $(\mathrm{pH}<2)$ là điều kiện tiên quyết để hòa tách vi sinh để giữ kim loại (cụ thể là sắt) trong dung dịch (Brandl và nnk., 2001)

Quy trình hòa tách vi sinh thích hợp để xử lý các nguyên liệu chứa hàm lượng kim loại thấp và ứng dụng ở quy mô nhỏ. Tuy nhiên, hầu hết các ứng dụng của phương pháp này dung để tái chế kim loại 
từ WEEE vẫn đang ở quy mô nghiên cứu phòng thí nghiệm.

\section{4. Định hướng công nghệ ở Việt Nam}

Theo số liệu điều tra của Tổng cục Thống kê, tỷ lệ các hộ gia đình Việt Nam sở hữu các đồ điện - điện tử gia dụng đang ngày càng tăng cao. So sánh tỷ lệ các hộ gia đình sử dụng EEE trong 2 năm 2004 và 2012: tỷ lệ số hộ sở hưu ti vi từ 0,698 chiếc/hộ lên 0,973 chiếc/hộ; tủ lạnh tăng 0,166 chiếc/hộ đến 0,947 chiếc/hộ; máy giặt từ 0,062 chiếc/hộ lên 0,227 chiếc/hộ (Huy., 2017). Theo nghiên cứu của URENCO (2007), ước tính năm 2010 có khoảng 217.187 tivi, 2,8 triệu điện thoại, 400.000 tủ lạnh, 100.000 điều hòa nhiệt độ và 542.000 máy giặt sẽ bị thải bỏ. Năm 2020, ước tính có 1,4 triệu tivi, 3,5 triệu điện thoại, 2,3 triệu tủ lạnh, 873.000 điều hòa nhiệt độ và hơn 2,6 triệu máy giặt sẽ bị thải bỏ (Duc và nnk; 2009).

Tuy nhiên, Việt Nam chưa có một quy định riêng về xử lý chất thải điện - điện tử mà chỉ quản lý như chất thải rắn thông thường. Các chất thải điện tử, điện gia dụng được coi là chất thải nguy hại được quy định trong Thông tư số 36/2015/TT BTNMT ngày 30 tháng 6 năm 2015 của Bộ trưởng Bộ Tài nguyên và Môi trường và được quản lý theo các quy định tại Luật Bảo vệ môi trường số 55/2014/QH13 ngày 23/6/2014, Nghị định $38 / 2015 / N D$ - CP ngày 24/4/2015 về quản lý chất thải và phế liệu. Do đó, vấn đề lớn nhất khiến Việt Nam chưa có ngành công nghiệp tái chế rác thải điện tử thật sự nằm ở việc thiếu đầu vào ổn định và đủ lớn. Việt Nam chưa có hệ thống quản lý để kiểm soát lượng lớn rác thải điện tử và thu hồi vật liệu có giá trị. Hiện nay, các cơ sở trung gian - những người tháo dỡ vật liệu - đang nhận nhiều lợi ích kinh tế nhất, nhưng họ không xử lý mà chỉ tháo dỡ để bán. Việc thu gom rác thải điện tử được thực hiện chủ yếu bởi các cá nhân làm nghề đồng nát, sửa chữa thiết bị hoặc các trung tâm, đại lý rác, các công ty môi trường đô thị. Do vậy, Nhà nước khó kiểm soát dòng rác thải điện tử và nguồn rác thải không tập trung, doanh nghiệp không thể đầu tư công nghệ tái chế hiện đại khi đầu vào thấp và không ổn định.

Đến nay, chỉ có Tổ chức Việt Nam tái chế (VRP) gồm các nhà sản xuất thiết bị điện và điện tử hàng đầu như: HP Inc, Apple, Microsoft đã nỗ lực thu gom miễn phí rác thải điện tử nhằm bảo đảm quy trình tái chế sản phẩm điện tử chuyên nghiệp và thân thiện môi trường. Tuy nhiên, thách thức lớn nhất mà tổ chức này đang phải đối mặt là người tiêu dùng không thuận lợi và còn khá ngại mang thiết bị điện tử đến chương trình vì cho rằng VRP không mang lại cho họ lợi ích.

Với hiện trạng thực tế ở trong nước và các công nghệ tái chế kim loại từ WEEE trên thế giới, nhận thấy rằng để giảm thiểu ô nhiễm từ nguồn rác thải điện - điện tử ngày càng tăng, Việt Nam cần đưa ra cơ chế quản lý thu gom và phân loại rác thải này, phải xây dựng các bãi thải riêng và từ đó có kế hoạch hỗ trợ các doanh nghiệp tái chế rác thải điện - điện tử. Công nghệ tái chế phù hợp hiện nay cho việc tái chế kim loại từ rác thải điện tử trong nước là công nghệ thủy luyện. Đây là quy trình dễ tiến hành, phù hợp ở quy mô vừa và nhỏ, vốn đầu tư thấp, dễ điều khiển và ít ô nhiễm môi trường.

\section{Kết luận}

Rác thải điện - điện tử đang là nguồn rác thải có tốc độ phát sinh lớn nhất ở các nước Châu Á nói chung và các nước trên thế giới nói riêng. Mặt khác, rác thải điện tử đang là dòng rác thải được luân chuyển từ các nước phát triển đến các nước đang phát triển và kém phát triển. Rác thải điện tử cũng là loại rác thải chứa nhiều kim loại quý và cả các chất độc hại có thể gây nguy hiểm cho môi trường nếu sử dụng công nghệ tái chế không phù hợp. Do vậy, việc ứng dụng các quy trình xử lý để thu hồi kim loại trong rác thải điện tử là một yêu cầu mang tính cấp thiết và có tính ứng dụng thực tiễn cao.

Phân tách vật lý là một phương pháp tái chế đầy hứa hẹn mà không gây ô nhiễm môi trường với đầu tư thiết bị hợp lý, chi phí năng lượng thấp và cho sản phẩm đa dạng. Tuy nhiên, việc tách phần kim loại và phi kim loại ra khỏi chất thải PCB chưa thật sự hiệu quả và đặc biệt không thể tách được các kim loại quý hiếm. Quá trình này thường chỉ sử dụng trong khâu đầu tiên trong công nghệ tái chế kim loại từ WEEE.

Tái chế hóa học thông qua các phương pháp luyện kim hỏa luyện và thủy luyện đều cho hiệu quả cao và có thể thu hồi được các kim loại quý. Quy trình hỏa luyện có ưu điểm ngắn gọn, năng suất cao, phù hợp ở quy mô lớn, do đó cần phải có nguồn nguyên liệu đủ và ổn định. Tuy nhiên, phương pháp này có nhược điểm là sinh ra khí độc như điôxin, nên cần phải có hệ thống xử lý phức tạp, đắt tiền. Quy trình thủy luyện hiện nay với các dung môi hòa tách clo và amoniac - amoni thân thiện môi trường đang được quan tâm phát triển và có nhiều triển 
vọng trong việc tái chế kim loại từ WEEE.

Tái chế vi sinh là quá trình mới, quy trình đơn giản, chi phí thấp và thân thiện môi trường nhất. Tuy nhiên phương pháp này mói bước đầu nghiên cứu trong phòng thí nghiệm để tách vàng và đồng, chưa đề cập tới các nguyên tố kim loại khác có trong WEEE. Do đó, phương pháp này cần phải trải qua một khoảng thời gian nghiên cứu nhất định nữa mói có khả năng triển khai vào thực tiễn.

\section{Những đóng góp của tác giả}

Tác giả Trần Trung Tới hình thành ý tưởng, cấu trúc bài báo và hoàn thiện bản thảo cuối cùng; tác giả Vũ Thị Chinh hoàn thiện mục 2; tác giả Phạm Thị Nhung hoàn thiện mục 4.

\section{Tài liệu tham khảo}

Akcil, A., Erust, C., Gahan, C. S., Ozgun, M., Sahin, M., Tuncuk, A., (2015). Precious metal recovery from waste printed circuit boards using cyanide and non - cyanide lixiviants e a review. Waste Manag. 45, 258 - 271.

Brandl, H., Bosshard, R., Wegmann, M., (2001). Computer - munching microbes: metal leaching from electronic scrap by bacteria and fungi. Hydrometallurgy 59, 319 - 326.

Behnamfard, A., Salarirad, M. M., Veglio, F., (2013). Process development for recovery of copper and precious metals from waste printed circuit boards with emphasize on palladium and gold leaching and precipitation. Waste Manag. 33, 2354 - 2363.

Cui, J., Forssberg, E. (2003). Mechanical recycling of waste electric and electronic equipment: a review. Journal of Hazardous Materials B99, 243 $-263$.

Dalrymple, I., Wright, N., Kellner, R., Bains, N., Geraghty, K., Goosey, M., Lightfoot, L. (2007). An integrated approach to electronic waste (WEEE) recycling. Circuit World 33 (2), 52 - 58.

Duan, C., Wen, X., Shi, C., Zhao, Y., Wen, B., He, Y. (2009). Recovery of metals from waste printed circuit boards by a mechanical method using a water medium. Journal of Hazardous Materials $166(1), 478-482$.

Duc, Quang Nguyen, Eiji Yamasue, Hideyuki Okumura, Keiichi N. Ishihara (2009). Use and disposal of large home electronic appliances in Vietnam. Journal of Material Cycles Waste Management, Vol. 11, No.4: pp 358 - 366.

Galbraith, P., Devereux, J. L. (2002). Beneficiation of printed wiring boards with gravity concentration. In: IEEE International Symposium on Electronics \& the Environment, 6 - 9 May, pp. 242 - 248.

Gurung, M., Adhikari, B. B., Kawakita, H., Ohto, K., Inoue, K., Alam, S., (2013). Recovery of gold and silver from spent mobile phones by means of acidothiourea leaching followed by adsorption using biosorbent prepared from persimmon tannin. Hydrometallurgy 133, 84 - 93.

Ha, V. H., Lee, J. C., Jeong, J., Hai, H. T., Jha, M. K., (2010). Thiosulfate leaching of gold from waste mobile phones. J. Hazard. Mater. 178, 1115 1119

He, Y., Xu, Z., (2015). Recycling gold and copper from waste printed circuit boards using chlorination process. RSC Adv. 5, 8957 - 8964.

Huy, Trần Đức, (2017). Nghiên cứu quy trình công nghệ thu hồi đồng từ bảng mạch điện tử thải bằng phương pháp hỏa luyện kết hợp điện phân. Đề tài Khoa học và Công nghệ cấp bộ, mã số: B2016 - BKA - 30

Koyama, K., Tanaka, M., Lee, J. - c., (2006). Copper leaching behavior from waste printed circuit board in ammoniacal alkaline solution. Mater. Trans. 47, 1788 - 1792.

Li, J., Zeng, X., Chen, M., Ogunseitan, O.A., Stevels, A., (2015). "Control - Alt - Delete": rebooting solutions for the e - waste problem. Environ. Sci. Technol. 49, 7095 - 7108.

Li, J., Shrivastava, P., Gao, Z., Zhang, H. C., (2004). Printed circuit board recycling: a state - of the art survey. IEEE Transactions on Electronics Packaging Manufacturing 27 (1), 33 - 42.

Li, J., Safarzadeh, M. S., Moats, M. S., Miller, J. D., LeVier, K. M., Dietrich, M., Wan, R. Y., (2012b). Thiocyanate hydrometallurgy for the recovery of gold. Part I: chemical and thermodynamic considerations. Hydrometallurgy 113 - 114, 1 - 9.

Lim, Y., Lee, J., Yoo, K., (2013). The ammonia leaching of alloy produced from waste printed 
circuit boards smelting process. Geosystem Eng. 16, $216-224$.

Liu, R., Shieh, R. S., Yeh, R. Y. L., Lin, C. H., (2009). The general utilization of scrapped PC board. Waste Manag. 29, 2842 - 2845

Moskalyk, R. R., Alfantazi, A. M., (2003). Review of copper pyrometallurgical practice: today and tomorrow. Miner. Eng. 16, 893 - 919.

Paul T. Williams, (2010). Valorization of Printed Circuit Boards from Waste Electrical and Electronic Equipment by Pyrolysis, Waste Biomass Valor 1:107 - 120.

Sun, Z. H. I., Xiao, Y., Sietsma, J., Agterhuis, H., Visser, G., Yang, Y., (2015). Selective copper recovery from complex mixtures of end - of - life electronic products with ammonia - based solution. Hydrometallurgy 152, 91 - 99.

Urban Environmental and Resource Cooperation (URENCO). Report on the Development of Ewaste Inventory in Vietnam, 2007.

Vanessa Forti, Cornelis Peter Baldé, Ruediger Kuehr, Garam Bel, (2020). The Clobal E-waste Monitor 2020. ISBN Print: 978-92-808-9115-7.

Xue Wang, Gabrielle Gaustad, (2012). Prioritizing material recovery for end - oflife printed circuit boards, Waste Management 321903 - 1913
Yang, X Y., Moats, M. S., Miller, J. D., Wang, X. M., Shi, X. C., Xu, H., (2011). Thioureaethiocyanate leaching system for gold. Hydrometallurgy 106, $58-63$.

Yazıcı, E. Y., Deveci, H., (2009). Recovery of metals from $\mathrm{E}$ - wastes. The Journal of the Chamber of Mining Engineers of Turkey 48 (3), 3 - 18

Yazici, E. Y., Deveci, H., (2013). Extraction of metals from waste printed circuit boards (WPCBs) in $\mathrm{H}_{2} \mathrm{SO}_{4}-\mathrm{CuSO}_{4}-\mathrm{NaCl}$ solutions. Hydrometallurgy 139, 30 - 38.

Yazici, E. Y., Deveci, H., (2015). Cupric chloride leaching $\left(\mathrm{HCl}-\mathrm{CuCl}_{2}-\mathrm{NaCl}\right)$ of metals from aste printed circuit boards (WPCBs). Int. J. Miner. Process. 134, 89 - 96.

Yoo, J.M., Jeong, J., Yoo, K., Lee, J., Kim, W., (2009). Enrichment of the metallic components from waste printed circuit boards by a mechanical separation process using a stamp mill. Waste Management 29, 1132 - 1137

Zhang, S., Forssberg, E., (1997). Mechanical separation - oriented characterization of electronic scrap. Resources, Conservation and Recycling 21, 247 - 269.

Zhao, Y., Wen, X., Li, B., Tao, D., (2004). Recovery of copper from printed circuit boards. Minerals and Metallurgical Processing 21 (2), 99 - 102. 